\title{
A SIMO one-bit time reversal for UWB communication systems
}

\author{
Dariush Abbasi-Moghadam ${ }^{{ }^{*}}$ and Vahid Tabataba Vakili
}

\begin{abstract}
With the one-bit time reversal ultra wideband (OTR-UWB) transceiver, the data symbols are encoded using the reversed order of the channel phase. The major factor limiting SISO OTR capacity and performance is intersymbol interference (ISI). As the data rate goes up, the ISI becomes more severe and it degrades system performance and capacity. In this article, a new single input multiple output (SIMO) system is proposed for an OTR-UWB system. The proposed transceiver structure is based on spatial focusing property of OTR. The performance of the proposed SIMO OTR-UWB system is analyzed in terms of the signal-to-interference-plus-noise-ratio (SINR). It is shown that using a SIMO OTR transceiver, ISI is reduced and the system capacity is increased almost linearly with the number of received antenna. Transmitted signal power at SIMO OTR decreases therefore in low data rates, SISO performance is better than SIMO, but in high rate scenario, SIMO OTR suppresses ISI better than SISO OTR and its performance is better. It is possible to compensate the reduced power using a receiver with more sensitivity, but to compensate the ISI effects, an MMSE receiver or equalizer techniques should be used, since the computational complexity of MMSE receiver grows exponentially with channel length and equalizers reduce the efficient bit rate. It is shown that the proposed SIMO-OTR capacity linearly increases with the number of antennas and decreases logarithmic (almost linearly).
\end{abstract}

Keywords: one-bit time reversal, UWB, SIMO, SINR, capacity

\section{Introduction}

For applications like sensor networks, ultra-low power, low complexity transmission schemes are of primary importance. UWB transceivers are a promising candidate technology, in particular because of their promise of providing high precision range and position information as well. UWB also has many attractive properties, including low interference to and from other wireless systems, low sensitivity to fading, easier wall-and floor penetration and high performance. However, large number of resolvable paths and low power limitations necessitate a complex receiver system. The receiver is usually implemented using a Rake with nearly 100 or more correlators in order to maintain acceptable signal-to-noise ratio (SNR) by gathering sufficient signal energy [1]. Rake receivers are further burdened with the problem of estimating the amplitude and the delay of each

\footnotetext{
* Correspondence: Abbasi@ee.iust.ac.ir

${ }^{1}$ Electrical Engineering Department, Shahid Bahonar University of Kerman, Kerman, Iran

Full list of author information is available at the end of the article
}

multipath component. Due to these problems, there is an impellent need for simpler receiver structures, capable of exploiting the rich UWB multipath channel diversity at the affordable cost, reasonable power consumption, and low complexity. Because of complexity constraints in practice, time reversal UWB receivers, one-bit time reversal, energy detector receiver and transmitted reference (or autocorrelation receiver) receiver are employed. Autocorrelation receiver and energy detector receivers do not require a channel estimation section. Autocorrelation receiver requires a long delay line that is very difficult to be implemented in hardware. Noncoherent energy detection receivers can serve these needs without expensive channel estimation and RAKE filters. The energy detector has a complexity advantage in the sense that no coherent carrier recovery is needed. But, it typically loses some $5 \mathrm{~dB}$ or more of SNR with respect to optimal coherent receivers [1-4]. Using time reversal, extremely simple noncoherent receivers can be used which have low-cost and need low-power. Given specific time and location, TR precoding has 
mathematically been proven to be optimum in the sense that it maximizes the amplitude of the field at that time and location [2]. Due to the spatial and temporal focusing properties of the TR, some problems in communication systems such as intersymbol interference (ISI) and multi-user interference can be alleviated. Even though TR can achieve the full multipath diversity, it is somehow difficult to implement in real-world UWB systems [3]. The TR complexity increases along with the length of the pre-filters and the quantization steps used in the channel estimation process. It is practically very difficult to deploy the pre-filter TR with a very fine quantization steps in rate of several gig symbol per second. Several attempts have been made to reduce the time reversal complexity using only the phase of the channel impulse response (CIR), the so-called one-bit time reversal, to construct the pre-filter [5]. In other word, the transmit filters in one-bit time reversal preserve only the sign information of the CIR. One-bit time reversal ultra wideband (OTR-UWB; also known as channel phase precoding UWB) has shown promising results for improving the system performance $[5,6]$. To overcome the shortcomings of TR-based UWB systems, CPP UWB transceiver architecture was proposed and showed that the spatial property of time reversal remained, but its delay spread increased [7]. Since the OTR system demands the receiver to estimate and feedback the signs of channel tap coefficients only (rather than the complete CIR), its complexity is significantly lower than that of time reversal. At the transmitter end, a more expensive linear amplifier is needed in time reversal to provide a larger signal dynamic range as compared with OTR $[5,8]$. As the data rate goes up, the ISI of OTR system becomes more severe and it degrades system performance more than background noise. We show that using a single input multiple output (SIMO) OTR transceiver, ISI is reduced and the system capacity is increased almost linearly with the number of received antenna. According to authors' knowledge, no research work about SIMO OTR-UWB system has been reported so far. In this article, such a system is proposed in order to decrease ISI and improve the system capacity.

At first, a SIR metric for SISO OTR-UWB system is defined and derived, based on UWB channel parameters. The receiving antenna of proposed SIMO-OTR should not be placed in spatial focusing depth of each other. It is shown that by approving a limited level of ISI, it is possible to increase the data rate with a low complexity receiver. In this article, SNR and signal-to-interferenceplus-noise-ratio (SINR) of SISO OTR-UWB system are derived analytically. Afterward, a SIMO OTR-UWB scheme is presented to mitigate ISI effects and its performance is analyzed and simulated. Then, capacity of SIMO OTR-UWB system is calculated and shows that
SIMO-OTR capacity is almost linearly increased with the number of antennas.

The structure of the article is as follows: the principle of OTR UWB is introduced in Section 2. Then SISO OTR-UWB ISI power is analyzed in Section 3. Transceiver structure of SIMO OTR-UWB and simulation and numerical results of its performance are presented in Section 4. Capacity analysis is discussed in Section 5. Finally, we conclude the article in Section 6.

\section{OTR-UWB communication system}

An OTR-UWB system uses the sign of CIR from the transmitter to the receiver as a transmitted pre-filter. Therefore, the received signal is

$$
y(t)=\underbrace{x_{s}(t) \otimes \operatorname{sign}\left(h(-t)^{*}\right)}_{\text {Transmitted Signal after OTR }} \otimes \underbrace{h(t)}_{\text {CIR }}+\underbrace{n(t)}_{\text {Noise }}
$$

where $\otimes$, " denote convolution and complex conjugate, respectively, $h(t)$ is the CIR and $x_{s}(t)$ is the transmitted signal before OTR.

Figure 1 shows the structure of SISO OTR transceiver. It is shown that the received signal is focused on the space at the intended receiver. Through spatial focusing, an OTR-UWB system is capable of mitigating cochannel interference effectively [5,7]. Figure 2 shows the simulation results for equivalent impulse response of TR-UWB and OTR-TR UWB system, as it is seen, the equivalent impulse response of OTR-UWB is not symmetric. It was observed that the effective channel becomes longer and poses a strong peak after precoding. It has been shown previously that one-bit time reversal in a multiple scattering or reverberating medium gives a higher peak-tonoise ratio than a classical TR because it gives more importance to the longest scattering paths, thus artificially reinforcing multiple scattering.

It was shown that if the delay spread of the original channel is $15.65 \mathrm{~ns}$, then the delay spread of time reversal and OTR is 16.43 and 28.02 ns, respectively (near CM3 channel) [7]. Due to delay spread increasing in OTR-UWB, its ISI power increases, thus the received signal must be equalized. In fact, the maximum data rate which may be sent before the time dispersion produces significant errors from ISI in the channel is related to channel RMS delay $\operatorname{Spread}\left(\tau_{\mathrm{RMS}}\right)$ by $B_{c}=1 /$ $\left(5 \tau_{\text {RMS }}\right)[4,9]$. Hence, for CM3 and CM1 OTR-UWB, the maximum data rate is about 8 and $22.4 \mathrm{Mbps}$, respectively.

The carrierless, tap-delayed line channel model is adopted. Thus, the CIR of UWB channel can be written as

$$
h(t)=\sum_{i=0}^{L-1} h_{i} \delta(t-i \Delta)=\sum_{i=0}^{L-1} p_{i} \alpha_{i} \delta(t-i \Delta)
$$




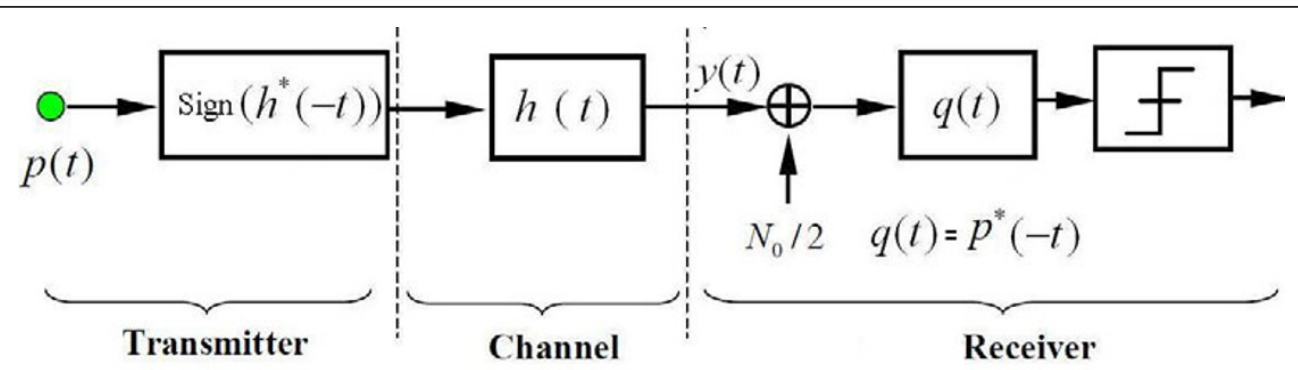

Figure 1 One-bit time reversal for UWB communication system.

where $L$ is the total number of paths, $\Delta$ is the multipath resolution that is assumed to be the same as the time domain pulse width, $h_{i}=p_{i} \alpha_{i}, p_{i} \in\{-1,+1\}$ is the phase of the $i$ th path with an equal probability, and $\alpha_{i}$ is the corresponding magnitude which is modeled as an independent Rayleigh random variable with probability density function $f_{\alpha_{i}}(x)=\frac{x}{\sigma_{i}^{2}} e^{-x^{2} / 2 \sigma_{i}^{2}}$. The first moment of $\alpha_{i}$ is $\sqrt{\frac{\pi}{2} \sigma_{i}^{2}}=\frac{\sqrt{\pi \gamma^{i}}}{2}$ and its second and fourth moments are $2 \sigma_{i}^{2}=\gamma^{i}, 8 \sigma_{i}^{4}=2 \gamma^{2 i}$, respectively, where
$\gamma=e^{-\Delta / \Gamma}$ and $\Gamma$ is the mean RMS delay spread, and the average power of $\alpha_{0}$ is 1 .

The transmitted signal after OTR [5]

$$
x(t)=\left[\sum_{i=0}^{L-1} p_{i} \alpha_{i} \delta(t-i \Delta)\right] \otimes \operatorname{sign}\left(h^{*}(-t)\right) \otimes p(t)=p(t) \otimes h_{\mathrm{eq}}(t)
$$

where $p(t)=x_{s}(t)$ is transmitted signal before OTR. Equivalent CIR $\left(h_{\mathrm{eq}}(t)\right)$ could be written as

$$
h_{e q}(t)=\sum_{i=0}^{2 L-2} h_{i}^{e q} \delta(t-i \Delta)
$$
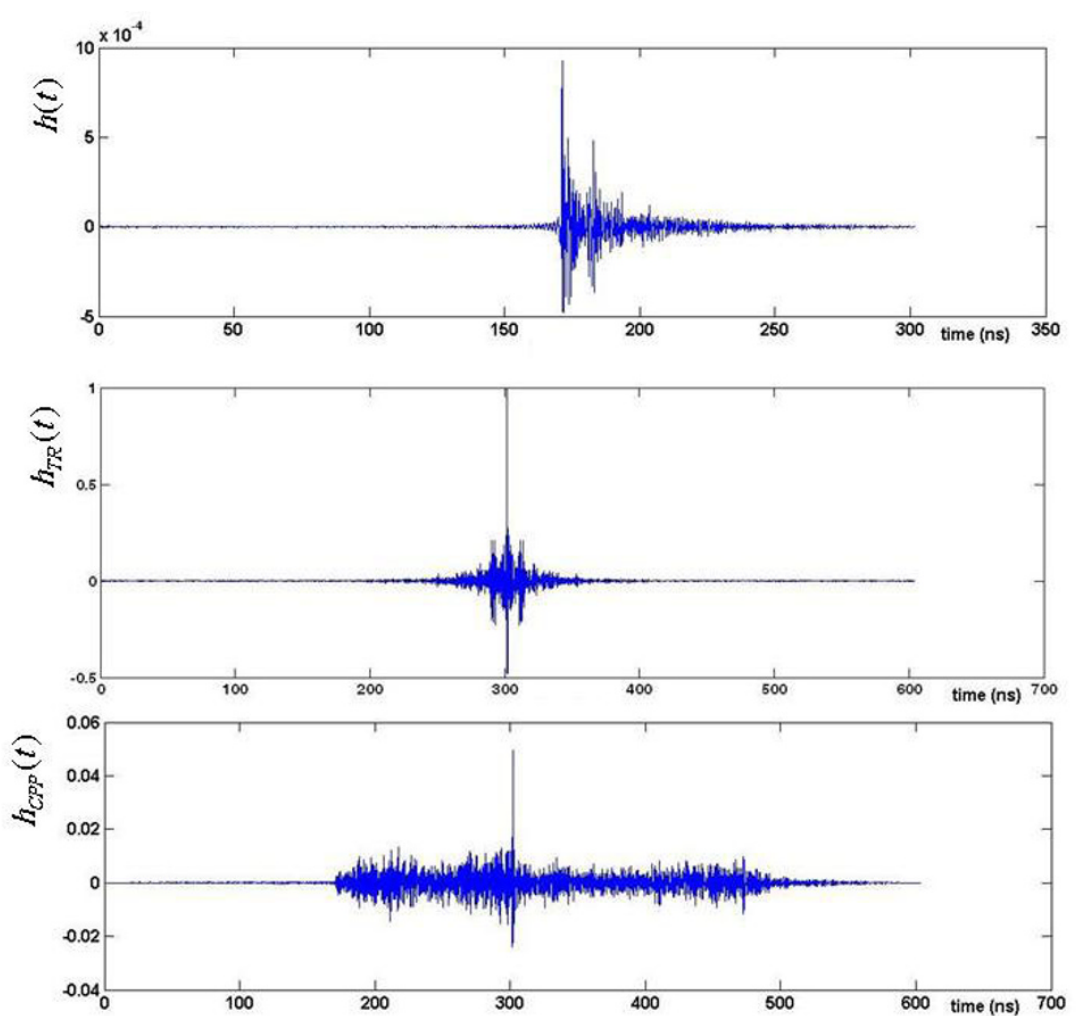

Figure 2 CIR of UWB, TR-UWB, and one-bit time reversal UWB system. 
Based on the definition of convolution, $h_{i}^{\mathrm{eq}}$ is

$$
h_{i}^{e q}=\left\{\begin{array}{cl}
\sum_{j=0}^{i} h_{j} p_{L-1+j-i} & 0 \leq i \leq L-1 \\
\sum_{j=i-L+1}^{L-1} h_{j} p_{L-1+j-i} & L \leq i \leq 2 L-2
\end{array}\right.
$$

If in the second term $\mathrm{k}=j-i+L-1$ and replacing $h_{i}$ in (5), $h_{i}^{\mathrm{eq}}$ is

$$
h_{i}^{e q}=\left\{\begin{array}{c}
\sum_{j=0}^{i} p_{j} \alpha_{j} p_{L-1+j-i} \quad 0 \leq i \leq L-1 \\
\sum_{k=0}^{2 L-2+i} p_{k+i-L+1} p_{k} \alpha_{k+i-L+1} \quad L \leq i \leq 2 L-2
\end{array}\right.
$$

The received power is

$$
\begin{aligned}
& S=P_{0} E\left(h_{L-1}^{e q}\right)^{2}=P_{0} E\left(\sum_{j=0}^{L-1} \alpha_{j} p_{j} p_{L-1+j-j-(L-1)}\right)^{2}=P_{0} E\left(\sum_{j=0}^{L-1} \alpha_{j} p_{j} p_{j}\right)^{2} \\
& =P_{0} E\left(\sum_{j=0}^{L-1} \alpha_{j}\right)^{2}=P_{0}\left(\sum_{j=0}^{L-1} E\left(\alpha_{j}^{2}\right)+\sum_{j=0}^{L-1} \sum_{\substack{i=0 \\
i \neq j}}^{L-1} E\left(\alpha_{i} \alpha_{j}\right)\right)=P_{0}\left(\sum_{j=0}^{L-1} \gamma^{j}+\frac{\pi}{4} \sum_{j=0}^{L-1} \sum_{\substack{i=0 \\
i \neq j}}^{L-1} \gamma^{(i+i)) / 2}\right) \\
& =P_{0}\left(\frac{1-\gamma^{L}}{1-\gamma}+\frac{\pi}{4}\left[\left(\sum_{i=0}^{L-1} \gamma^{i / 2}\right)^{2}-\sum_{i=0}^{L-1} \gamma^{i}\right]\right)=\frac{P_{0} \pi}{4}\left[\frac{(4-\pi)\left(1-\gamma^{L}\right)}{(1-\gamma)}+\left(\frac{1-\gamma^{L / 2}}{1-\gamma^{1 / 2}}\right)^{2}\right]
\end{aligned}
$$

where $E$ denotes expectation and $P_{0}$ is the transmitted signal power.

For a non-ISI scenario, SNR is

$$
\mathrm{SNR}=\frac{P_{0} \pi}{4 P_{\mathrm{N}}}\left[\frac{(4-\pi)\left(1-\gamma^{L}\right)}{(1-\gamma)}+\left(\frac{1-\gamma^{L / 2}}{1-\gamma^{1 / 2}}\right)^{2}\right] \approx \frac{\pi P_{0}}{4 P_{\mathrm{N}}}\left(\frac{1-\gamma^{L / 2}}{1-\gamma^{1 / 2}}\right)^{2}
$$

where $P_{\mathrm{N}}$ is the noise power at receiver output.

\section{OTR-UWB interference analysis}

Interference analysis is an important topic in reviewing OTR-UWB features. Due to the extremely large bandwidths of UWB systems, received signals are usually corrupted by interferences. ISI affects the performance of a UWB receiver; therefore, it is necessary to examine the properties of interference. A complete description of interference in terms of probability density function is difficult. However, derivation of the statistical moments of interference is possible if the symbol interval is shorter than the CIR length, the receiver output contains ISI.

We define an ISI metric which is the desired signal-tointerference-power ratio. As mentioned previously, because of nonsymmetric CIR, precursor ISI (ISI $\mathrm{pr}_{\mathrm{pr}}$ ) and post-cursor $\left(\mathrm{ISI}_{\mathrm{po}}\right)$ ISI are not the same. Therefore, the SIR is

$$
\mathrm{SIR}=\frac{S}{\mathrm{ISI}_{\mathrm{pr}}+\mathrm{ISI}_{\mathrm{po}}}
$$

Using uncorrelated scattering it can be shown that [5]

$$
\operatorname{ISI} \mathrm{Pr}_{\mathrm{pr}}=P_{0} E\left(\sum_{i=M}^{L-1}\left(h_{i}^{e_{i}}\right)^{2}\right)=P_{0} E\left(\sum_{j=1}^{L_{0}} h_{L-1-j M}^{2}\right)=P_{0} \sum_{j=1}^{L_{0}} \sum_{i=0}^{L-1-j M} E\left(\alpha_{i}^{2}\right)=P_{0} \sum_{j=1}^{L_{0}} \frac{1-\gamma^{L+j j M}}{1-\gamma}
$$

where $T_{s}=M \Delta$ is the symbol interval, $L_{0}=\lfloor(L-1) / M\rfloor$ and $\lfloor x\rfloor$ are the floor function of $x$. And similarly postcursor ISI is [5]

$$
\mathrm{ISI}_{\mathrm{po}}=P_{0} \sum_{j=1}^{L_{0}} \frac{1-\gamma^{L-j M}}{1-\gamma}
$$

As it is known, data rate $(R)$ is proportion to $1 / T_{s}$. Therefore, by increasing rate, $L_{0}$ increases and consequently ISI increases with data (symbol) rate increment.

The performance of OTR-UWB system in terms of output SNR and SINR is analyzed. The returned phase information is assumed to be perfect, in the following analysis. For a SISO OTR-UWB with binary pulse amplitude modulation signaling, SIR and SINR are

$$
\begin{aligned}
& \operatorname{SIR} \approx \frac{\frac{P_{0} \pi}{4}\left[\frac{(4-\pi)\left(1-\gamma^{L}\right)}{(1-\gamma)}+\left(\frac{1-\gamma^{L / 2}}{1-\gamma^{1 / 2}}\right)^{2}\right]}{P_{0} \sum_{j=1}^{L_{0}}\left\{\frac{1-\gamma^{L-j \mu}}{1-\gamma}+\frac{1-\gamma^{L+\mu}}{1-\gamma}\right\}}=\frac{\frac{\pi}{4}\left[\frac{(4-\pi)\left(1-\gamma^{L}\right)}{(1-\gamma)}+\left(\frac{1-\gamma^{L / 2}}{1-\gamma^{1 / 2}}\right)^{2}\right]}{\sum_{j=1}^{L}\left\{\frac{1-\gamma^{L-j / M}}{1-\gamma}+\frac{1-\gamma^{L+\mu}}{1-\gamma}\right\}} \\
& =\frac{\frac{\pi(1-\gamma)}{4}\left[\frac{(4-\pi)\left(1-\gamma^{L}\right)}{(1-\gamma)}+\left(\frac{1-\gamma^{L / 2}}{1-\gamma^{1 / 2}}\right)^{2}\right]}{\sum_{j=1}^{L}\left\{2-\gamma^{L-j M}-\gamma^{L / j M}\right\}}=\frac{\frac{\pi(1-\gamma)}{4}\left[\frac{(4-\pi)\left(1-\gamma^{L}\right)}{(1-\gamma)}+\left(\frac{1-\gamma^{L / 2}}{1-\gamma^{1 / 2}}\right)^{2}\right]}{2 L_{0}-\gamma^{L} \sum_{j=1}^{L}\left\{\gamma^{-j M}+\gamma^{j M}\right\}} \\
& =\frac{\frac{\pi(1-\gamma)}{4}\left[\frac{(4-\pi)\left(1-\gamma^{L}\right)}{(1-\gamma)}+\left(\frac{1-\gamma^{L / 2}}{1-\gamma^{1 / 2}}\right)^{2}\right]}{2 L_{0}-\gamma^{L} \sum_{j=0}^{L_{0}}\left\{\gamma^{j-j M}+\gamma^{j M}\right\}}=\frac{\frac{\pi\left(1+\gamma^{1 / 2}\right)\left(1-\gamma^{L / 2}\right)^{2}}{4\left(1-\gamma^{1 / 2}\right)}+\frac{\pi(4-\pi)}{4}\left(1-\gamma^{L}\right)}{2 L_{0}-\frac{\left.\left(1-\gamma^{L} L^{L}+\right)^{M}\right) \gamma^{L}}{1-\gamma^{M}}-\frac{\left(1-\gamma^{-\left(L_{0}+1\right) M} \gamma^{L}\right.}{1-\gamma^{-M}}} \\
& \operatorname{SINR}=\frac{S}{P_{N}+I S I_{p r}+I S I_{p o}} \approx \frac{\frac{P_{0} \pi}{4}\left[\frac{(4-\pi)\left(1-\gamma^{L}\right)}{(1-\gamma)}+\left(\frac{1-\gamma^{L / 2}}{1-\gamma^{1 / 2}}\right)^{2}\right]}{P_{N}+P_{0} \sum_{j=1}^{L_{0}}\left\{\frac{1-\gamma^{L-j M}}{1-\gamma}+\frac{1-\gamma^{L+j M}}{1-\gamma}\right\}}
\end{aligned}
$$

By simplifying (13), we have

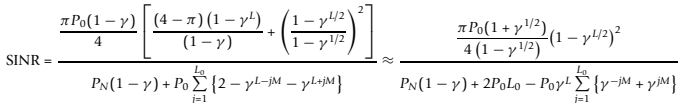

$$
\begin{aligned}
& =\frac{\frac{\pi P_{0}\left(1+\gamma^{1 / 2}\right)}{4\left(1-\gamma^{1 / 2}\right)}\left(1-\gamma^{L^{L / 2}}\right)^{2}}{P_{N}(1-\gamma)+2 P_{0} L_{0}-P_{0} \frac{\left(1-\gamma^{\left(L_{0}+1\right) M}\right) \gamma^{L}}{1-\gamma^{M}}-P_{0} \frac{\left(1-\gamma^{-\left(L_{0}+1\right) M} \gamma^{L}\right.}{1-\gamma^{-M}}}
\end{aligned}
$$

\section{Transceiver structure of proposed SIMO OTR- UWB}

In this section, the transceiver structure of a proposed SIMO OTR-UWB system is described. As discussed in previous section, OTR-UWB data rate is limited due to ISI effect; therefore, its performance and capacity are degraded. We would like the ISI to be as low as possible and the system capacity to be as high as possible. The system capacity can be increased by using a SIMO structure. A SIMO-OTR system configuration is illustrated in Figure 3. Let $h_{i}(t)$ denotes the CIR between antennas at the transmitter and the $i$ th antenna at the receiver, $p(t)$ is pulse shaping function, and $\operatorname{sign}\left(h_{i}^{*}(-t)\right)$ is the corresponding prefilter code employed in the $i$ th antenna branch at the transmitter. In this method, data are transmitted by a transmitter 


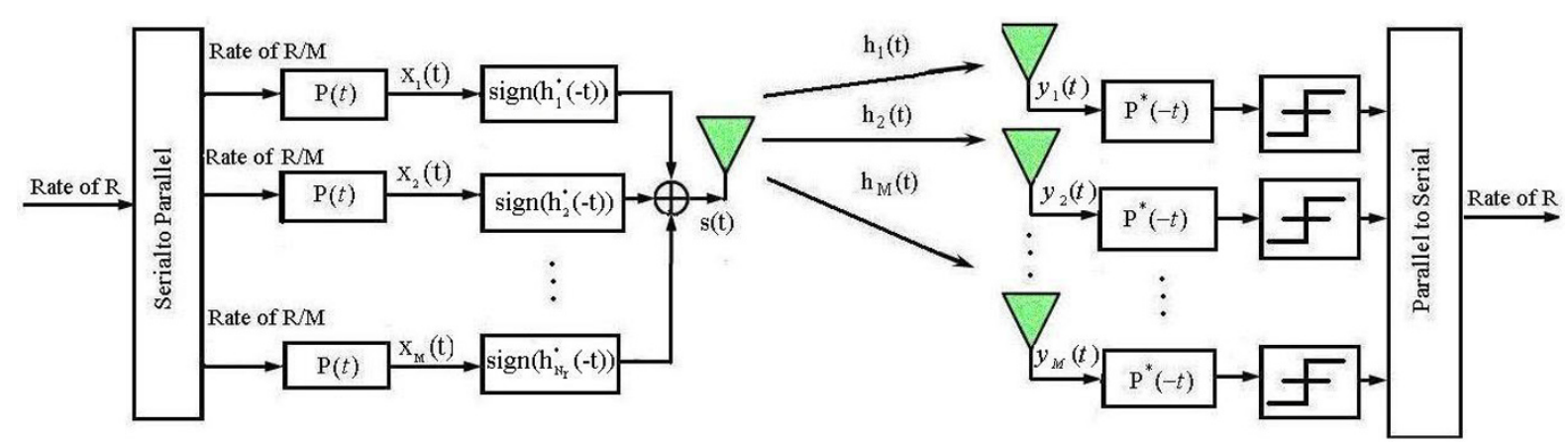

Figure 3 Block diagram of proposed SIMO OTR-UWB scheme.

comprising of a single antenna and received by multiple antennas. The transceiver structure is based on spatial focusing property of OTR-UWB system. One-bit TR retains the spatial property of classic time reversal [7]. In office environment, this directivity dropping $(20 \mathrm{~dB})$ can be achieved by setting the spacing between any two adjacent antenna elements greater than $20 \mathrm{~cm}$, which corresponds to the half wavelength of the lowest frequency [2]. In rectangular metal cavity environment, directivity of time reversal drops by $20 \mathrm{~dB}$ when the antenna is $5 \mathrm{~cm}$ away from the intended receiver [8]. Low spatial focusing gain of OTR-UWB system at distance $r$ away from the intended receiver indicates that a nearby receiver at that location would not be able to detect the signal. The receiving antenna should not be placed in spatial focusing depth of each other, therefore the channels $\left(h_{1}(t), h_{2}(t), \ldots, h_{N_{r}}(t)\right)$ are almost uncorrelated.

Data rate and power in each branch of transmitter decrease to $R / N_{r}$ and $P_{0} / N_{r}$, respectively, where $N_{r}$ is the number of received antenna. Therefore, the ISI power in the proposed scheme reduces due to lower rate (greater symbol interval) and the symbols can successfully be decoded by simple sampling the received signal at the appropriate instance.

The transmitted signal in proposed SIMO OTR-UWB is

$$
\begin{aligned}
s(t) & =x_{1}(t) \otimes \operatorname{sign}\left(h_{1}^{*}(-t)\right)+x_{2}(t) \otimes \operatorname{sign}\left(h_{2}^{*}(-t)\right)+\cdots+x_{N_{r}}(t) \otimes \operatorname{sign}\left(h_{N_{f}}^{*}(-t)\right) \\
& =\sum_{i=1}^{N_{f}} x_{i}(t) \otimes \operatorname{sign}\left(h_{i}^{*}(-t)\right)
\end{aligned}
$$

And the output of $l$ th antenna at the receiver is denoted by

$$
\begin{aligned}
y_{l}(t) & =h_{l}(t) \otimes \sum_{i=1}^{N_{r}} x_{i}(t) \otimes \operatorname{sign}\left(h_{i}^{*}(-t)\right)+n(t) \\
& =\underbrace{x(t) \otimes h_{l}(t) \otimes \operatorname{sign}\left(h_{1}^{*}(-t)\right)}_{\text {Signal }}+\underbrace{h_{l}(t) \otimes \sum_{i=1, i \neq}^{N_{r}} x_{i}(t) \otimes \operatorname{sign}\left(h_{i}^{*}(-t)\right)}_{\text {Cochannel interference }}+\underbrace{n(t)}_{\text {Noise }}
\end{aligned}
$$

$y_{l}(t)=x(t) \otimes h_{l}(t) \otimes \operatorname{sign}\left(h_{1}^{*}(-t)\right)+i_{s}(t)+n(t)=x(t) \otimes h_{\mathrm{eq}}(t)+i_{s}(t)+n(t)$

where $i_{s}(t)$ denotes cochannel interference part in (16). As it is seen the output of each branch in SIMO OTRUWB transceiver is similar to a SISO OTR-UWB system, but the power and rate of each branch at transmitter is decreased and an interfering term is added to each of them. With the constant transmitted power $P_{0}$, the associated power for each transmitter branch of SIMO OTR-UWB reduces to $P_{0} / N_{r}$. There is usually a power scaling factor included in the OTR code for SIMO to make sure that the transmit power remain the same, after OTR precoding. Without loss of generality, we suppose that this scaling factor is equal to 1 .

If the rate of each branch in transmitter is less than coherence bandwidth $\left(R / N_{r}<B_{c}\right)$, the ISI does not occur. Therefore, the received SNR of proposed SIMO-OTR is

$$
\mathrm{SNR}=\frac{P_{0} \pi}{4 N_{r} \times\left(P_{N}+I_{s}\right)}\left[\frac{(4-\pi)\left(1-\gamma^{L}\right)}{(1-\gamma)}+\left(\frac{1-\gamma^{L / 2}}{1-\gamma^{1 / 2}}\right)^{2}\right]
$$

Comparing (18) and (14) in rate higher than coherence bandwidth shows that the SIMO OTR performance is better than SISO OTR if ISI $\mathrm{pr}+\mathrm{ISI}_{\mathrm{po}} \geq\left(N_{r}-1\right) P_{N}$. In the other world, in low transmitted power, the ISI power is very less than noise power and the noise power is the dominant term $\left(\mathrm{ISI}_{\mathrm{pr}}+\mathrm{ISI}_{\mathrm{po}} \ll\left(N_{r}-1\right) P_{N}\right)$. Consequently, the proposed SIMO technique performs better than conventional SISO at higher values of $P_{0} / N_{0}$ and data rate.

It is possible to compensate the reduced power using a receiver with more sensitivity. But, more sensitive receiver cannot compensate for the ISI effect and in presence of ISI, equalizer or MMSE receiver should be used. The optimal receiver is the maximum likelihood sequence estimator (MLSE), since the computational complexity grows exponentially with channel length. Most channels of practical interest require too much computation for MLSE to be feasible. Therefore, suboptimal schemes like equalizers should be used to compensate for the ISI 
effects. Traditional equalizers use training sequences to adjust the tap weights. However, using training sequences decrease bandwidth efficiency.

We define a quality factor $(9)$ for rates greater than coherence bandwidth $\left(R / N_{r}<B_{c}, R>B_{c}\right)$ ISI condition, which is the ratio of SIMO SNR to SISO SINR:

$$
\begin{aligned}
\vartheta & =\frac{\text { SNR }_{\text {SIMO }}}{\text { SINR }_{\text {SISO }}} \approx \frac{\frac{P_{0} \pi}{4 N_{r} \times\left(P_{N}+I_{s}\right)}\left[\frac{(4-\pi)\left(1-\gamma^{L}\right)}{(1-\gamma)}+\left(\frac{1-\gamma^{L / 2}}{1-\gamma^{1 / 2}}\right)^{2}\right]}{\frac{\pi P_{0}(1-\gamma)}{4}\left[\frac{(4-\pi)\left(1-\gamma^{L}\right)}{(1-\gamma)}+\left(\frac{1-\gamma^{L / 2}}{1-\gamma^{1 / 2}}\right)^{2}\right]} \\
& =\frac{\frac{1 /\left(N_{r} \times\left(P_{N}+I_{s}\right)\right)}{(1-\gamma)}}{P_{N}(1-\gamma)+2 P_{0} L_{0}-P_{0} \frac{\left(1-\gamma^{\left(L_{0}+1\right) M}\right) \gamma^{L}}{1-\gamma_{0}} \frac{\left(1-\gamma^{-\left(L_{0}+1\right) M}\right) \gamma^{L}}{1-\gamma^{-M}}} \\
& =\frac{P_{N}(1-\gamma)+2 P_{0} L_{0}-P_{0} \frac{\left(1-\gamma^{\left(L_{0}+1\right) M}\right) \gamma^{L}}{1-\gamma^{M}}-P_{0} \frac{\left(1-\gamma^{-\left(L_{0}+1\right) M}\right) \gamma^{L}}{1-\gamma^{-M}}}{N_{r} \times\left(P_{N}+I_{s}\right)(1-\gamma)} \\
& =\frac{P_{N}}{N_{r}\left(P_{N}+I_{s}\right)}+\frac{\left(1-\nu_{0}\right.}{P_{r} \times(1-\gamma)\left(P_{N}+I_{s}\right)}\left[2 L_{0}-\frac{\left(1-\gamma^{\left(L_{0}+1\right) M}\right) \gamma^{L}}{1-\gamma^{M}}-\frac{\left(1-\gamma^{-\left(L_{0}+1\right) M}\right) \gamma^{L}}{1-\gamma^{-M}}\right]
\end{aligned}
$$

From (19) it is seen that for $\gamma<1$ and $L, M \gg 1, L$ $>M$, the quality factor is

$$
\vartheta \approx \frac{P_{N}}{N_{r}\left(P_{N}+I_{s}\right)}+\frac{P_{0}}{N_{r} \times(1-\gamma)\left(P_{N}+I_{s}\right)}\left[2 L_{0}-\frac{\gamma^{-\left(L_{0}+1\right) M+L}}{\gamma^{-M}-1}\right]
$$

For high $P_{0} /\left(P_{N}+I_{s}\right)$, the quality factor is

$$
\vartheta \approx \frac{P_{0}\left[2 L_{0}-\frac{1}{\gamma^{-M}-1}\right]}{N_{r} \times(1-\gamma)\left(P_{N}+I_{s}\right)}
$$

As it is seen from (20) and (21), 9 increases by increment of $P_{0} /\left(P_{N}+I_{s}\right)$ and $L_{0}$. But increment of the received antenna number decreases the quality factor because of power reduction in each branch of SIMO transmitter. The reduced power can be compensated using a receiver with more sensitivity.

For distance larger than $3 \mathrm{~m}, I_{s}$ is negligible (Appendix A), if $\gamma<1$ and $L \gg 1$, the SNR, SINR and $\vartheta$ of SIMOOTR is

$$
\begin{aligned}
& \mathrm{SNR}=\frac{\pi P_{0}}{4 N_{r} P_{N}}\left[\frac{(4-\pi)}{(1-\gamma)}+\frac{1}{\left(1-\gamma^{1 / 2}\right)^{2}}\right] \\
& \vartheta \approx \frac{1}{N_{r}}+\frac{P_{0}\left(2 L_{0}-1 /\left(\gamma^{-M}-1\right)\right)}{N_{r} \times(1-\gamma) P_{N}}
\end{aligned}
$$

If the received antennas do not placed in focusing depth, the interfering term in (13) is considerable. Similar to previous section, the power of this interfering part with consideration of directivity factor $\left(d_{f}\right)$ is

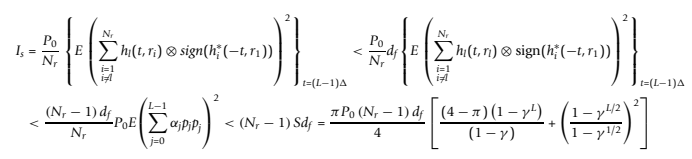

where $h_{i}\left(t, r_{i}\right)$ is the CIR between transmitter and receiver located in $r_{1}$.

It is possible to accept a limited level of ISI, data rate of each branch greater than coherence bandwidth, and design the system with lower complexity (smaller $N_{r}$ ). In this case, the received SINR of the proposed SIMOOTR scheme is

$$
\begin{aligned}
& \operatorname{SINR}=\frac{\frac{\pi P_{0}(1-\gamma)}{4 N_{r}}\left[\frac{(4-\pi)\left(1-\gamma^{L}\right)}{(1-\gamma)}+\left(\frac{1-\gamma^{L / 2}}{1-\gamma^{1 / 2}}\right)^{2}\right]}{\left(P_{N}+I_{s}\right)(1-\gamma)+\left(P_{0} / N_{r}\right) \sum_{j=1}^{L / N}\left\{2-\gamma^{L-j M}-\gamma^{L+j M}\right\}}=\frac{\frac{\pi P_{0}(1-\gamma)}{4}\left[\frac{(4-\pi)\left(1-\gamma^{L}\right)}{(1-\gamma)}+\left(\frac{1-\gamma^{L / 2}}{1-\gamma^{1 / 2}}\right)^{2}\right]}{\left(P_{N}+I_{s}\right)(1-\gamma) N_{r}+P_{0} \sum_{j=1}^{L_{N}}\left\{2-\gamma^{L-j M}-\gamma^{L+j M}\right\}} \\
& \approx \frac{\frac{\pi P_{0}\left(1+\gamma^{1 / 2}\right)}{4\left(1-\gamma^{1 / 2}\right)}\left(1-\gamma^{L / 2}\right)^{2}}{\left(P_{\mathrm{N}}+I_{S}\right)(1-\gamma) N_{\mathrm{r}}+2 P_{0} L_{0 l}-\frac{P_{0}\left(1-\gamma^{\left(L_{0}+1\right) M}\right) \gamma^{L}}{1-\gamma^{M}}-\frac{P_{0}\left(1-\gamma^{-\left(L_{\left.L^{+}+1\right) M}\right) \gamma^{L}}\right.}{1-\gamma^{-M}}}
\end{aligned}
$$

where $L_{O I}$ is the same as $L_{0}$ in limited ISI scenario.

In this case, it is possible to use of optimal one-bit time reversal UWB. In optimal OTR scheme, optimum number of taps is used to design the prefilter to obtain the best system performance. It is shown that in the temporal domain the performance of the one-bit TR system does not necessary improve when the number of prefilter coefficients increases [7,9]. Since the prefilter length selection criteria is based on the output SIR maximization, which is a highly nonlinear function of code rate (or symbol interval). It was observed that the optimal code length is the same as the symbol interval in the low rate scenario. However, in the high rate scenario, the optimal code length is just equal to multiple symbol intervals [6]. Therefore, due to lower rate of each branch in proposed SIMO-OTR scheme, its required prefilter length is smaller than SISO-OTR scheme. This means simple prefilter and smaller rat in feedback link for tap coefficients.

\subsection{Analytical and simulation results}

In this section, performance of SISO and proposed SIMO OTR-UWB systems are evaluated. The most widely adopted UWB multipath channel model has been proposed by the IEEE 802.15.3a Task Group [10]. Therefore, the CIR is simulated (generated) according to IEEE 802.15.3a channel model. This channel model is designed for SISO scenario; the extension to a SIMO scheme is achieved by assuming that the SIMO channel parameters are independent and identically distributed from the same statistical model. CM3 channel model is used in all simulation.

The performance of SISO OTR-UWB communication system in CM3 channel is shown in Figure 4, indicating a satisfying agreement between analytical and simulation results. Figure 5 shows the performance of SIMO OTRUWB communication system in bit rate of 50 Mbps. As it is seen in SISO OTR the maximum received SINR is about $15 \mathrm{~dB}$ and for high $P_{0} / N_{0}$ quality factor is very high. By increasing $P_{0} / N_{0}$ in Equation $17, P_{0} / N_{0} \gg 1$ or $P_{0} / N_{0} \rightarrow \infty$, it could be seen that the SINR is not 


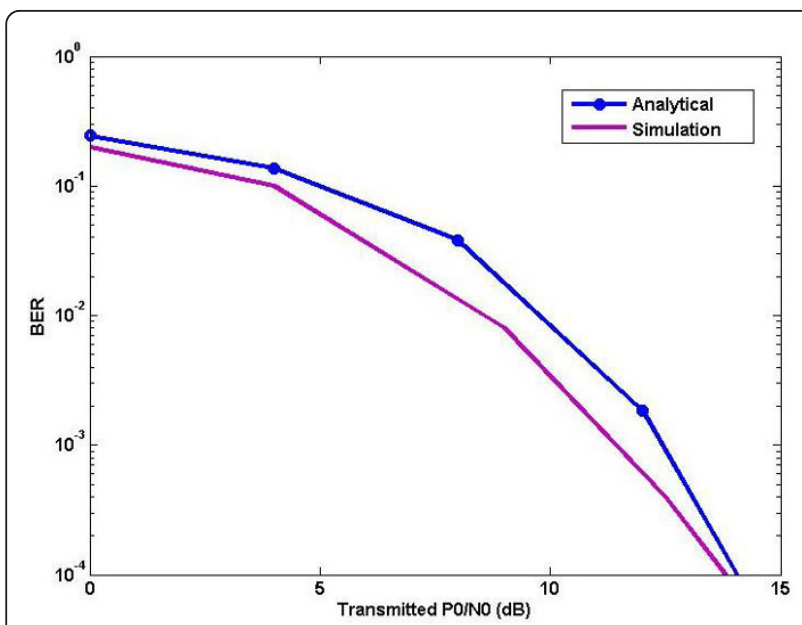

Figure 4 Performance of SISO OTR-UWB communication system in CM3 channel with $R=12$ Mbps.

increased and limited to SIR. Therefore, the performance is not improved by increasing $P_{0} / N_{0}$ and so the system capacity is limited. But, for proposed SIMO, the performance depends on $P_{0} /\left(N_{0} N_{r}\right)$ and more increasing $P_{0} / N_{0}$ results in more performance improvement. Figure 6 shows the bit error rate (BER) of SIMO and SISO OTR-UWB system in a CM3 UWB channel at data rate of 50 and $25 \mathrm{Mbps}$. As it is seen for a certain $P_{0} / N_{0}$, SIMO OTR performance is better than SISO OTR. As it is observed in BER of $10^{-4}$, SIMO-OTR with $N_{r}=3$ rate and of $25 \mathrm{Mbps}$ is $2 \mathrm{~dB}$ better than SISO; also the performance of SIMO-OTR with $N_{r}=6$ and rate of 50 Mbps is $1.8 \mathrm{~dB}$ better than SISO-OTR. Figure 7 shows that in higher SNR, the SIMO-OTR performance is better than SISO-OTR and in BER of $10^{-4}$, SIMO-OTR

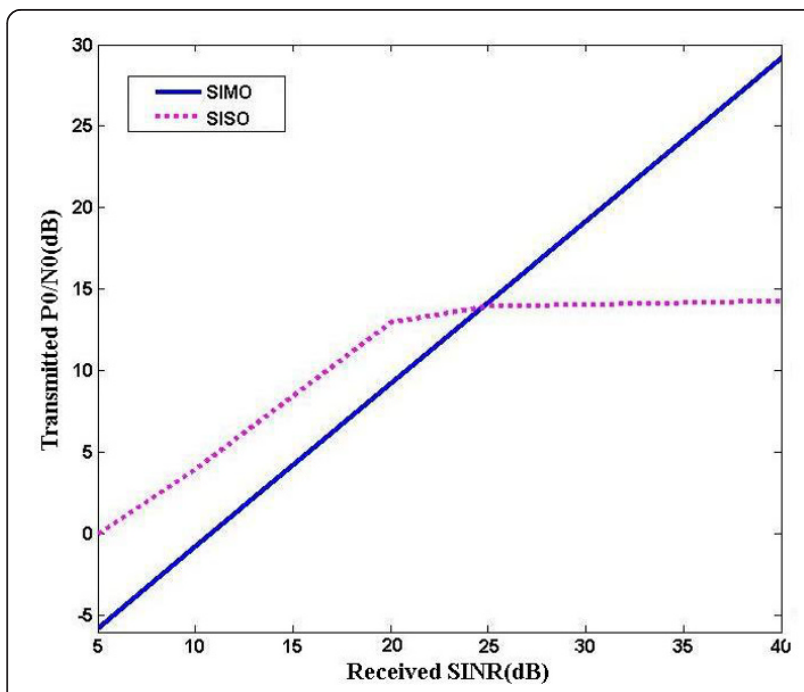

Figure 5 Performance of SISO\&SIMO OTR-UWB communication system in CM3 Channel with $R=50 \mathrm{Mbps}, N_{r}=6$.

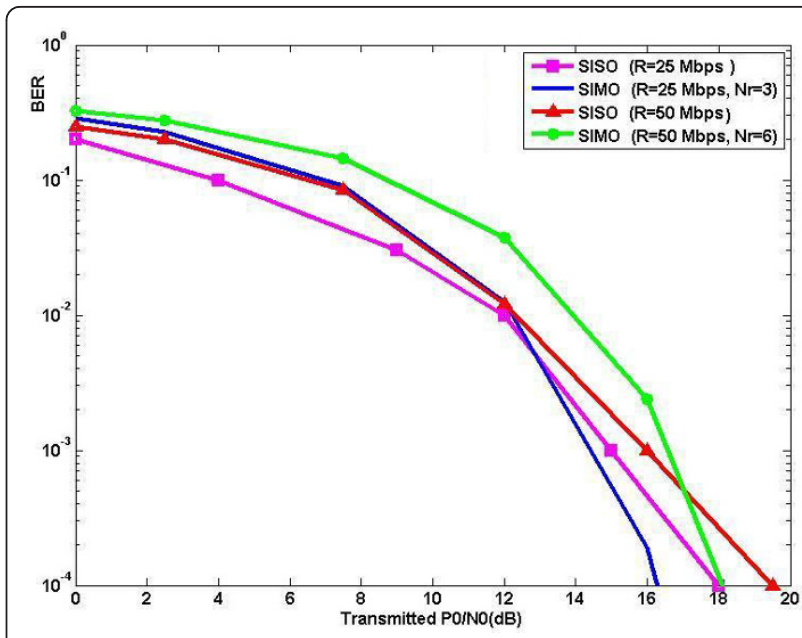

Figure 6 Simulated BER of SIMO and SISO OTR-UWB system in CM3 channel with $R=50$ Mbps.

with $N_{r}=2$ and rate of $50 \mathrm{Mbps}$ is $0.8 \mathrm{~dB}$ better than SISO-OTR with two transmitted antennas. Performance comparison of SIMO-OTR in BER of BER of $10^{-4}$ shows that the performance of SIMO-OTR with six received antennas is only $1 \mathrm{~dB}$ better than SIMO-OTR with two received antennas.

\section{Capacity analysis}

The channel capacity is an important parameter that can be used for determining tradeoff of wireless communication systems. The increase in data rate is not only required for long range communication, but also for short range communication. For single antenna systems, it is well known that given a fixed bandwidth, capacity can only be increased logarithmically with the SNR. UWB capacity is calculated for different spectrum-

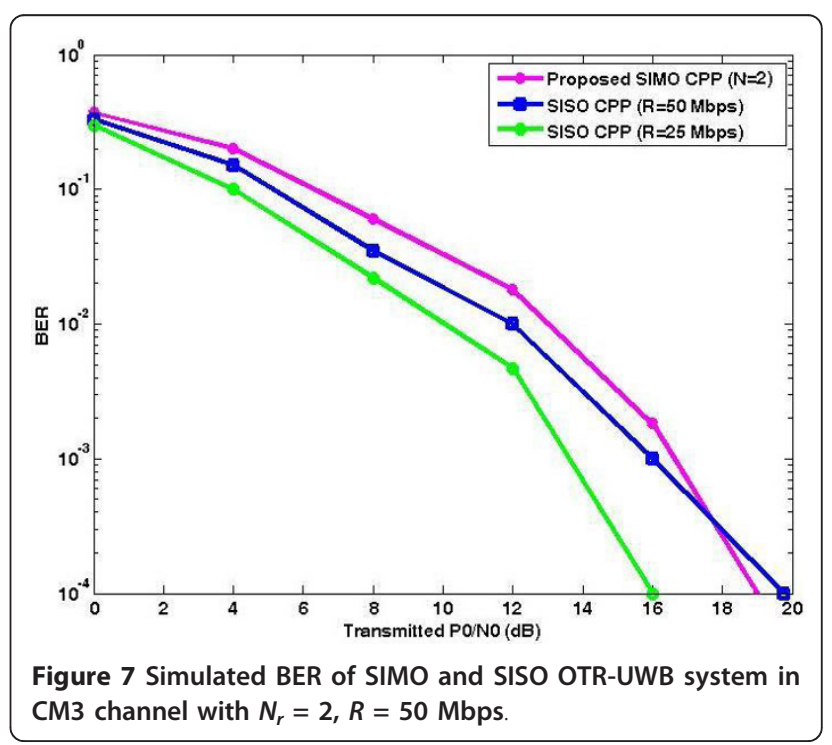


shaping schemes in [11]. It is shown that the capacity of SISO UWB scheme is

$$
C=\int_{f_{0}}^{f_{1}} \log _{2}\left(1+\frac{P_{0}|H(f)|^{2}}{N_{0}}\right) d f
$$

where $f_{0}, f_{1}$ are the starting and the end frequencies, respectively, $H(f)$ is the OTR equivalent channel transfer function, $P_{0}$ is the signal power at transmitter output and $N_{0}$ is the power spectral density (PSD) of noise.

And, the spectrum efficiency is

$$
\frac{C}{\mathrm{BW}}=\frac{1}{f_{1}-f_{0}} \int_{f_{0}}^{f_{1}} \log _{2}\left(1+\frac{P_{0}|H(f)|^{2}}{N_{0}}\right) d f
$$

where BW is bandwidth.

By considering a limited level of ISI, the capacity of SISO OTR-UWB scheme is

$$
C=\int_{f_{0}}^{f_{1}} \log _{2}\left(1+\frac{P_{0}|H(f)|^{2}}{N_{0}+I}\right) d f
$$

The SIMO-OTR splits the input serial data into $N_{r}$ parallel data streams that are transmitted simultaneously by prefiltering the transmitted signal and packing the multiple signals in the same time slot. Thus, the rate of the proposed SIMO-OTR is ameliorated by factor $N_{r}$ due to the $N_{r}$-parallel transmitted data in the channel. If the received antenna is not located in focusing depth, cochannel interference occurred, therefore the capacity of SIMO TR-UWB scheme is reduced to

$$
C \approx N_{r} \int_{f_{0}}^{f_{1}} \log _{2}\left(1+k \frac{P_{0}\left|H_{\mathrm{eq}-s}(f)\right|^{2}}{N_{r} \times N_{0}}\right) d f
$$

where $H_{\text {eq-s }}(f)$ is the equivalent SISO OTR transfer function and $k$ is the variable which takes into account the peak power loss due to cochannel interference. ${ }^{a}$

For comparison, the capacities of MISO and MIMO systems are [12]

$$
C \approx\left\{\begin{array}{lr}
\int_{f_{0}}^{f_{1}} \log _{2}\left(1+\frac{N_{r} \times P_{0}\left|H_{e q-s}(f)\right|^{2}}{N_{0}}\right) d f & \text { for MISO } \\
N_{r} \int_{f_{0}}^{f_{1}} \log _{2}\left(1+\frac{P_{0}\left|H_{e q-s}(f)\right|^{2}}{N_{0}}\right) d f & \text { for MIMO }
\end{array}\right.
$$

where $H_{\text {eq-s }}(f) \approx H_{1}(f) \approx H_{2}(f) \cdots \approx H_{N_{r}}(f)$ is the channel transfer function of equivalent SISO-OTR of SIMO scheme.
By considering a limited level of ISI, the capacity of SIMO OTR-UWB scheme is

$$
C \approx N_{r} \int_{f_{0}}^{f_{1}} \log _{2}\left(1+k \frac{P_{0}\left|H_{e q-s}(f)\right|^{2}}{N_{r} \times\left(N_{0}+I\right)}\right) d f
$$

As it is seen from (30) and (31), the proposed SIMO OTR capacity is better than classical SISO, standard SIMO, and MISO scenarios. Furthermore, its capacity is less than MIMO, but its decreasing is logarithmic. In other words, although the SIMO and MISO channels can only offer a logarithmic increase in capacity with the number of antennas [13], the proposed SIMO-OTR capacity is linearly increased with the number of antennas and decreased logarithmic (approximately linear); therefore, the main advantage of proposed SIMO structure is capacity increment.

Figure 8 shows the relative capacity of SIMO TRUWB to the rate of SISO TR-UWB system. It shows that the data rate increases significantly with $N_{r}$ for a constant SNR especially for high SNR. For instance, for a SNR of $30 \mathrm{~dB}$, the data rate increases by a factor of 3.2 for $N_{r}=4$ and 5.6 for $N_{r}=8$.

\section{Conclusion}

To reduce the time reversal UWB complexity, a one-bit time reversal UWB scheme is used in which the transmit prefilters preserve only the sign information of the CIR. As the data rate increases, the ISI of OTR system becomes more severe and it degrades system performance and capacity. A SIMO structure is proposed to reduce ISI in higher data rates. In this article, we first derived SINR for

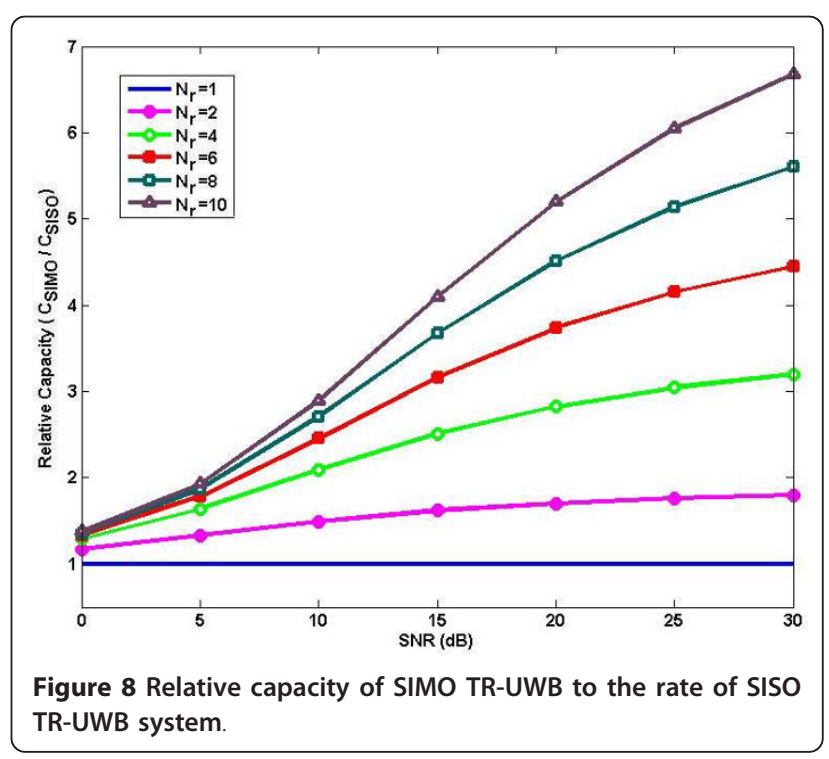


a one-bit time reversal UWB system based on UWB channel parameters. It was shown that in low data rate, SISO OTR performance is better than SIMO because of lower ISI and higher received SNR. In higher rates, SIMO OTRUWB is better than SISO OTR-UWB because its ISI is much less than SISO OTR. It was seen that in data rate of $6 R_{c}$ and $P_{0} / N_{0}$ of $30 \mathrm{~dB}$ performance of SIMO OTR with $N_{r}=6$ is $4 \mathrm{~dB}$ better than SISO TR, where $N_{r}$ is the number of received antenna. This improvement enhanced in higher rates with increasing the receiving antennas. The main advantage of SIMO OTR compared with SISO and MISO is its higher capacity. It was shown that SIMO capacity is $N_{r}$ times more than SISO OTR.

\section{Appendix A: Cochannel interference}

The FCC requires that the maximum PSD of UWB system be $-41.3 \mathrm{dBm} / \mathrm{MHz}$. We considered the impulse radio signal which fully covers the FCC band [14], i.e., 3.1-10.6 GHz. The center frequency was set to be $f_{0}=$ $6.85 \mathrm{GHz}$. Path loss is

$$
\text { Path loss }=\left(\frac{\lambda}{4 \pi d}\right)^{n}=\left(\frac{c}{4 \pi f_{0} d}\right)^{n}
$$

where $n$ depends on the propagation environment. It was shown that $1.4<n<4.1$ for a regular indoor environment except for the hard-NLOS situation [15]. In office environment, $n=1.92$; with obstructions (NLOS), $n=3.633[16]$.

Office environment path loss in $d=1 \mathrm{~m}$ from transmitter and LOS propagation is $-49.15 \mathrm{~dB}$. If the directivity $(d r)$ drops by $20 \mathrm{~dB}$ at unintended receiver and minimum antenna number of $2\left(N_{r}=2\right)$ then the maximum PSD at unintended received antenna, $P_{r}$, is

$$
P_{r}<-41.3-\text { Path loss }-10 \log \left(N_{r} /\left(N_{r}-1\right)\right)-d r=-41.3-49.15-3-20=-113.45 \mathrm{dBm} / \mathrm{MHz}
$$

The PSD of the AWGN noise is $-114 \mathrm{dBm} / \mathrm{MHz}$, as it is seen in $d=1 \mathrm{~m}$, PSD of cochannel interference in LOS environment is almost the same as AWGN PSD. However, by increasing $d, M$, and $f_{0}$, PSD of cochannel interference is much less than PSD of AWGN, for example in $d=6 \mathrm{~m}, M=2$, and $f_{0}=6.85 \mathrm{GHz}, P_{r}$ is $-129 \mathrm{dBm} / \mathrm{MHz}$. Therefore, the cochannel interference in far field of transmitter is negligible.

\section{Endnote}

${ }^{\text {a }}$ Similar to Appendix A, it can be shown that at frequency of $6.85 \mathrm{GHz}$ and $N_{r}=2, k$ is equal to $0.5,0.8$, 0.98 in $d=1,2$, and $6 \mathrm{~m}$, respectively. Therefore, it could be concluded that in great distance $k \simeq 1$.

\section{Acknowledgements}

The authors would like to thank ITRC (Iran Telecommunication Research Center) for their invaluable assistance and funding for this research.

\section{Author details}

${ }^{1}$ Electrical Engineering Department, Shahid Bahonar University of Kerman, Kerman, Iran ${ }^{2}$ Department of Telecommunications, School of Electrical Engineering, Iran University of Science \& Technology (IUST), Narmak, Tehran, Iran

Received: 26 February 2011 Accepted: 20 March 2012

Published: 20 March 2012

\section{References}

1. MZ Win, RA Scholtz, Energy capture vs. correlator resources in ultra-wide bandwidth indoor wireless communications channels, in Proc IEEE Military Communications Conference (MILCOM'97), vol. 3. Monterey, CA, USA, pp. 1277-1281 (November 1997)

2. C Zhou, N Guo, B Sadler, RC Qiu, Performance study on time reversed impulse MIMO for UWB communications based on measured spatial UWB channels, in Military Communications Conference, MILCOM 2007. IEEE, Orlando, FL, USA, pp. 1-6 (29-31 October 2007)

3. D Abbasi-Moghadam, V Tabataba Vakili, Characterization of indoor time reversal UWB communication systems: spatial, temporal and frequency properties. Wiley Intersci J Commun Syst. doi:10.1002/dac.1140

4. D Abbasi-Moghadam, V Tabataba Vakily, Channel characterization of time reversal UWB communication systems. Springer Ann Telecommun. 65(9-10), 601-614 (2010). doi:10.1007/s12243-010-0189-8

5. Y-H Chang, S-H Tsai, X Yu, CCJ Kuo, Ultra wideband (UWB) transceiver design using channel phase precoding (CPP). IEEE Trans Signal Process. 55(Part 2, 7), 3807-3822 (2007)

6. HT Nguyen, Optimal one bit time reversal for UWB impulse radio in multiuser wireless communications. Int J Electron Commun Comput Eng. 1(3) (2009)

7. P Kyritsi, P Papanicolaou, One-bit time reversal for WLAN applications, in IEEE 16th International Symposium on Personal, Indoor and Mobile Radio Communications (PIMRC 2005), Berlin, Germany, pp. 532-536 (11-14 September 2005)

8. D Singh, Z Hu, RC Qiu, UWB channel sounding and channel characteristics in rectangular metal cavity, in IEEE SoutheastCon 08, Huntsville, Alabama, pp. 323-328 (3-6 April 2008)

9. Y-H Chang, S-H Tsai, X Yu, C-CJ Kuo, Design and analysis of channel-phaseprecoded ultra wideband (CPPUWB) systems, in IEEE Wireless Communications and Networking Conference, Las Vegas, Nevada, USA, pp. 866-871 (3-6 April 2006)

10. JR Foerster, Channel modeling sub-committee report (final). Tech Rep P802.15-02/368r5-SG3a, IEEE P802.15 Working Group for Wireless Personal Area Networks (WPANs) (December 2002)

11. RC Qiu, B Sadler, Z Hu, Time reversed transmission with chirp signaling for UWB communications and its application in confined metal environments, in IEEE International Conference on Ultra-Wideband, ICUWB 2007, Singapore, pp. 276-281 (24-26 September 2007)

12. RC Qiu, Z Hu, MIMO Capacity for UWB channel in rectangular metal cavity, in Proc of IEEE Southeastern Symposium, Huntsville, AL, USA, pp. 129-135 (36 April 2008)

13. D Gesbert, M Shafi, D Shan Shiu, PJ Smith, A Nagui, From theory to practice: an overview of MIMO space-time coded wireless systems. IEEE J Sel Areas Commun. 21(3), 281-302 (2003). doi:10.1109/JSAC.2003.809458

14. T Rappaport, Wireless Communications: Principles and Practice, 2nd edn. (Pearson Education Pte Ltd, 2002)

15. J Ahmadi-Shokouh, RC Qiu, Ultra-wideband (UWB) communications channel measurements-a tutorial review. Int J Ultra Wideband Commun Syst. 1(1), 11-31(21) (2009). doi:10.1504/IJUWBCS.2009.026447

16. W Ciccognani, A Durantini, D Cassioli, Time domain propagation measurements of the UWB indoor channel using PN-sequence in the FCCcompliant band $3.66 \mathrm{GHz}$. IEEE Trans Antenn Propag. 53(4), 1542-1549 (2005)

\section{doi:10.1186/1687-1499-2012-113}

Cite this article as: Abbasi-Moghadam and Vakili: A SIMO one-bit time reversal for UWB communication systems. EURASIP Journal on Wireless Communications

and Networking 2012 2012:113. 